\title{
Marcadores bioquímicos na COVID-19: Uma revisão na literatura
}

\author{
Biochemical markers in COVID-19. A literature review \\ Marcadores bioquímicos en COVID-19: Una revisión en la literatura
}

Recebido: 17/02/2021 | Revisado: 23/02/2021 | Aceito: 24/02/2021 | Publicado: 06/03/2021

\author{
Amanda Bócoli de Almeida \\ ORCID: https://orcid.org/0000-0003-3298-3737 \\ Universidade José do Rosário Vellano, Brasil \\ E-mail: amanda.balmeida@aluno.unifenas.br \\ Juan Salles Abrão \\ ORCID: https://orcid.org/0000-0003-3294-6039 \\ Universidade José do Rosário Vellano, Brasil \\ E-mail:juan.abrao@aluno.unifenas.br \\ Tânia Nogueira Luz \\ ORCID: https://orcid.org/0000-0002-2862-5999 \\ Universidade José do Rosário Vellano, Brasil \\ E-mail: Tania.luz@aluno.unifenas.br \\ Ana Carolina Lima Delmoro \\ ORCID: https://orcid.org/0000-0002-5827-080X \\ Universidade José do Rosário Vellano, Brasil \\ E-mail: carol_delmoro@outlook.com \\ Felipe Fidelis Camilo \\ ORCID: https://orcid.org/0000-0002-3812-4012 \\ Universidade José do Rosário Vellano, Brasil \\ E-mail: felipe.camilo@aluno.unifenas.br \\ Carlos Henrique Santos Oliveira \\ ORCID: https://orcid.org/0000-0003-4959-8149 \\ Universidade José do Rosário Vellano, Brasil \\ E-mail: carlos.unifenas@gmail.com \\ Franciele Souza \\ ORCID: https://orcid.org/0000-0002-3010-1254 \\ Universidade José do Rosário Vellano, Brasil \\ E-mail: francielemiranda52@outlook.com \\ Michele Caroline Terra \\ ORCID: https://orcid.org/0000-0003-4139-4256 \\ Universidade José do Rosário Vellano, Brasil \\ E-mail: michelecaroline_2012@hotmail.com \\ Carla Miguel de Oliveira \\ ORCID: https://orcid.org/0000-0003-2812-7647 \\ Universidade Federal de Alfenas, Brasil \\ E-mail: carla_oliveira03@hotmail.com \\ Bruno Cesar Correa Salles \\ ORCID: https://orcid.org/0000-0002-4444-9673 \\ Universidade José do Rosário Vellano, Brasil \\ E-mail: bruno.alfenas@hotmail.com
}

\begin{abstract}
Resumo
A pandemia causada pela COVID-19 apresentou diversos desafios para as comunidades médicas, de pesquisa e para os serviços públicos de saúde. Por esse motivo, é de extrema importância o conhecimento sobre o perfil bioquímico dos infectados, uma vez que ele poderá auxiliar no diagnóstico e tratamento de possíveis complicações. O objetivo do presente trabalho é realizar uma revisão da literatura capaz de demonstrar os marcadores bioquímicos de pacientes COVID-19 positivos ou negativos com base nos resultados dos exames de sangue de rotina de publicações. Para o seu desenvolvimento foi realizada uma busca eletrônica nas bases de dados Scielo, Pubmed, Science Direct e Google Scholar. Os resultados dessa revisão demonstraram a importância da amplificação do RNA viral por RT-PCR, pois pode ser utilizado como padrão para a confirmação da infecção que detecta o ácido nucleico. Todavia, são observados outros marcadores como limiares para AST e LDH que permitiram prever um prognóstico de complicações dos pacientes COVID-19-positivos ou negativos tendo como base os resultados da análise de sangue de rotina. O exame de sangue pode ser usado como uma alternativa para a RT-PCR no monitoramento do agravamento de pacientes COVID-19 positivos. As alterações bioquímicas ainda não estão totalmente elucidadas. Desse modo, conclui-se que é de extrema importância conhecer as alterações bioquímicas que possam estar associadas à infecção pelo vírus SARSCoV 2, podendo auxiliar no prognóstico da COVID-19 e escolha do melhor tratamento para possíveis complicações. Palavras-chave: COVID-19; RT-PCR; SARS-CoV; Marcadores bioquímicos; Proteína C reativa.
\end{abstract}




\begin{abstract}
The pandemic caused by COVID-19 presented several challenges for the medical, research and public health services communities. For this reason, knowledge about the biochemical profile of those infected is extremely important, since it can help in the diagnosis and treatment of possible complications. The aim of the present study is to perform a literature review capable of demonstrating the biochemical markers of positive or negative COVID-19 patients based on the results of routine blood tests in publications. For its development, an electronic search was performed in the databases Scielo, Pubmed, Science Direct and Google Scholar. The results of this review demonstrated the importance of amplifying viral RNA by RT-PCR, as it can be used as a standard for confirming the infection that detects nucleic acid. However, other markers are observed as thresholds for AST and LDH that allowed predicting a prognosis of complications in COVID-19-positive or negative patients based on the results of routine blood analysis. The blood test can be used as an alternative to RT-PCR in monitoring the worsening of COVID-19 positive patients. Biochemical changes are not yet fully understood. Thus, it is concluded that it is extremely important to know the biochemical changes that may be associated with infection by the SARS-CoV 2 virus, which can assist in the prognosis of COVID-19 and the choice of the best treatment for possible complications.
\end{abstract}

Keywords: COVID-19; RT-PCR; SARS-CoV; Biochemical markers; C-reactive protein.

\title{
Resumen
}

La pandemia causada por COVID-19 presentó varios desafíos para las comunidades de servicios médicos, de investigación y de salud pública. Por este motivo, el conocimiento del perfil bioquímico de los infectados es de suma importancia, ya que puede ayudar en el diagnóstico y tratamiento de posibles complicaciones. El objetivo del presente estudio es realizar una revisión de la literatura capaz de demostrar los marcadores bioquímicos de pacientes COVID19 positivos o negativos a partir de los resultados de los análisis de sangre de rutina en publicaciones. Para su desarrollo se realizó una búsqueda electrónica en las bases de datos Scielo, Pubmed, Science Direct y Google Scholar. Los resultados de esta revisión demostraron la importancia de amplificar el ARN viral mediante RT-PCR, ya que puede usarse como estándar para confirmar la infección que detecta ácido nucleico. Sin embargo, se observan otros marcadores como umbrales de AST y LDH que permitieron predecir un pronóstico de complicaciones en pacientes COVID-19 positivos o negativos en función de los resultados de los análisis de sangre de rutina. El análisis de sangre se puede utilizar como una alternativa a la RT-PCR para controlar el empeoramiento de los pacientes positivos a COVID-19. Los cambios bioquímicos aún no se comprenden completamente. Así, se concluye que es de suma importancia conocer los cambios bioquímicos que pueden estar asociados a la infección por el virus SARS-CoV 2, que pueden ayudar en el pronóstico del COVID-19 y en la elección del mejor tratamiento ante posibles complicaciones.

Palabras clave: COVID-19; RT-PCR; SARS-CoV; Marcadores bioquímicos; Proteína C-reactiva.

\section{Introdução}

Popularmente conhecido como Coronavírus, a Covid-19 (SARS-CoV-2) é uma doença que acomete principalmente o trato respiratório, podendo variar de quadros clínicos assintomáticos até formas mais graves, como insuficiência respiratória (Yuki et al., 2020). É o grande responsável pela síndrome respiratória aguda grave coronavírus (Serrano-Cumplido et al., 2020).

A Covid-19 trata-se de uma pneumonia que foi descoberta no início de dezembro de 2019 em Wuhan, uma cidade localizada na China, e se espalhou rapidamente por todo o mundo (Croda \& Garcia, 2020). O SARS-CoV 2 pertence à mesma família de vírus (Coronaviridae) que causou epidemias anteriores, como a síndrome respiratória do Oriente Médio (MERSCoV) em 2012 e a síndrome respiratória aguda coronavírus (SARS-CoV) em 2003 (Serrano-Cumplido et al., 2020).

A infecção pelo SARS-CoV 2 está ainda em curso de descoberta. Entretanto, o vírus possui a capacidade de se espalhar para todos os órgãos do corpo, podendo assim levar a alterações hematológicas, imunológicas e até mesmo bioquímicas. Segundo Lai et al., 2020, as alterações bioquímicas ainda não estão totalmente claras.

O surto de COVID-19 apresentou desafios para as comunidades médicas, pesquisa e para os serviços públicos de saúde, por isso que o perfil bioquímico de infectados é tão importante, pois serve para auxiliar em diagnósticos e tratamentos de possíveis complicações. Sendo assim, caracterizar os marcadores bioquímicos irá permitir prever um prognóstico de complicações dos pacientes COVID-19 positivos ou negativos com base nos resultados dos exames de sangue de rotina. A análise de sangue pode ser usada como uma alternativa para RT-PCR para monitorar o agravamento de pacientes COVID-19 positivos. O objetivo deste trabalho é realizar uma revisão de literatura para demonstrar os marcadores bioquímicos de 
pacientes COVID-19 positivos ou negativos com base nos resultados dos exames de sangue de rotina de publicações atuais.

\section{Metodologia}

O trabalho realizado não envolveu o uso de dados de seres humanos, não necessitando de qualquer tipo de avaliação clínico-laboratorial e, assim, não foi necessário a aprovação pelo Comitê de Ética. O estudo trata-se de uma pesquisa bibliográfica sistemática, constituída de livros e artigos científicos (Pereira et al. 2018). Foi efetuada uma revisão de literatura sobre o tema através de revistas acadêmicas científicas e artigos, através de bancos de dados, tais como Scielo, Pubmed, Science direct e Google Scholar, de modo que se comparou as diferentes informações encontradas nestas plataformas, listando as principais condições que aborda os marcadores bioquímicos na COVID-19. Foram pesquisados artigos utilizando os seguintes descritores: perfil bioquímico, COVID-19, marcadores hepáticos, marcadores renais, marcadores cardíacos, marcadores pancreáticos, marcadores biliares e SARS-COV 2. Os critérios de inclusão foram: artigos publicados entre 2000 e 2020, originais, em língua portuguesa e inglesa, que obedeciam às palavras chaves descritas. Foram excluídos editoriais e opiniões.

Figura 1: Esquematização dos métodos de busca/Figure 1: Layout of the search methods.

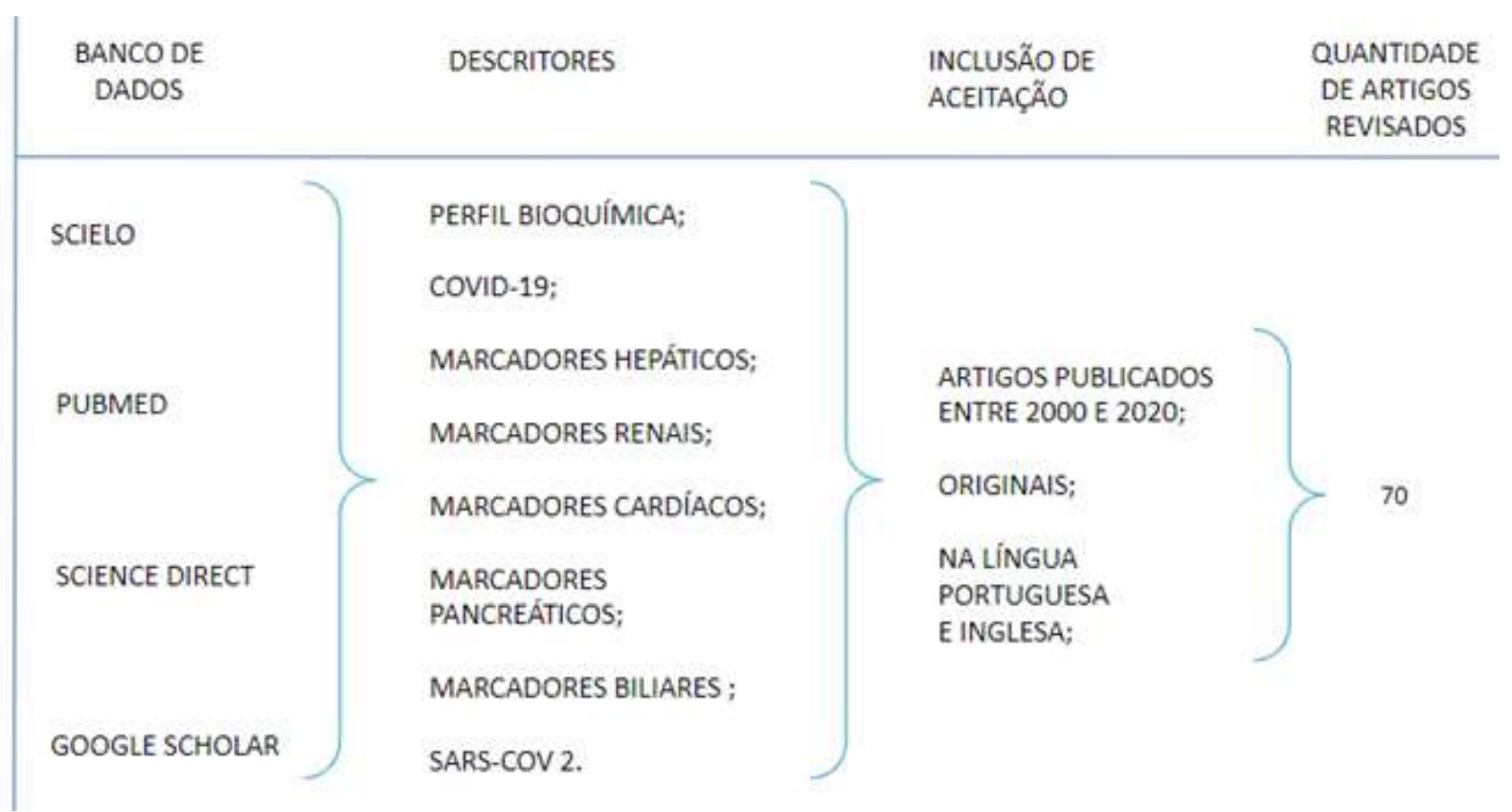

Fonte: Autores.

\section{Resultados e Discussão}

A recente doença do coronavírus humana pertencente à família viral Coronaviridae é o sétimo coronavírus entre os 14 tipos de vírus que são divididos entre os grupos alfa, beta, gama e delta. No século 21, o SARS-CoV e o MERS-CoV foram agentes de epidemias e o SARS-CoV-2 conhecido como a cepa mais recente e causadora da nova pandemia mundial (V. G. da Costa et al., 2020).

A doença COVID-19 foi comunicada pela primeira vez em Wuhan, China, logo após se espalhou por todo o mundo. O coronavírus foi designado como uma síndrome respiratória aguda grave coronavírus 2 (SARS-CoV-2) pelo Comitê Internacional de Taxonomia de vírus com base em análise filogenética (Yu, 2020). 
Três tipos de coronavírus foram registrados durante um período de 18 anos, como a síndrome respiratória aguda que ocorreu em 2002, e a síndrome respiratória do Oriente Médio em 2012, com o aparecimento do MERS (de Paula Antunes et al., 2020).

No dia 30 de janeiro de 2020, a OMS (Organização Mundial de Saúde) declarou a infecção pelo SARS-COV 2 uma pandemia. Ainda, de acordo com a OPAS, 2020, um levantamento de casos feito no dia 4 de janeiro de 2021 mostra 7.753 .752 casos de infecção pelo covid-19, sendo que 196.561 deles levaram a óbito.

A enzima conversora de angiotensina 2 (ACE2) é o principal sítio de ligação do vírus SARS-CoV-2, que é expressa no rim muito mais do que nos pulmões (Cui et al., 2020). É possível que o vírus entre no rim invadindo os podócitos, primeiro ganhando acesso ao líquido tubular e, subsequentemente, o vírus se liga ao ACE2 no túbulo proximal (Jia et al., 2005). A entrada do coronavírus nas células-alvo do hospedeiro também requer a agregação do envelope viral com as membranas celulares. Os peptídeos de SARS-CoV ativados por fusão são criados por clivagem proteolítica específica das proteínas S, em uma etapa chamada "priming". Como consequência, a infectividade celular não depende apenas da expressão de ACE2, mas também é governada por tipos de proteases encontradas em um determinado tipo de célula.

No rim é a enzima protease transmembrana serina 2 (TMPRSS2) que ativa a proteína SARS-CoV-2 S, que é expressa fortemente no néfron distal ao invés do túbulo proximal. Alternativamente, o tropismo de SARS-CoV-2 pode ser expandido pelo local de clivagem de furina exclusivo na proteína Spike que é processado durante a biogênese (Diao et al., 2020a).

\subsection{Diagnóstico Laboratorial e Alterações Bioquímicas na COVID -19}

Após três surtos de infecções por coronavírus humano $(\mathrm{HCoV})$ no início do século XXI, foi enfatizada a importância de testes para diagnóstico rapidamente disponíveis, precisos e rápidos. Mesmo sendo um desafio obter um diagnóstico preciso por conta da variabilidade e urgência na confirmação, obteve-se uma enorme evolução dos métodos a fim de limitar a propagação, bem como para tratar adequadamente esses pacientes (Loeffelholz \& Tang, 2020). Existem diversos tipos de marcadores tais como físicos (alterações características em estruturas biológicas), fisiológicos (funções de órgãos), histológicos (amostras de tecido obtidas por biópsia) e anatômicos podendo ser enzimas, hormônios, genes, moléculas ou células específicas, sendo os mais importantes para a investigação médica, aqueles que podem ser obtidos facilmente através de fluidos biológicos (Pinto, 2017). Os biomarcadores podem ser divididos em três tipos: de exposição, de efeito e de suscetibilidade, os quais são ferramentas que tornam possível a identificação de uma substância tóxica ou uma condição adversa antes que tenham indícios de danos à saúde (Amorim, 2003).

Os marcadores são a expressão bioquímica da lesão, os biomarcadores de exposição estimam a dose interna, por meio da determinação de uma substância química ou de seu produto gerado através da biotransformação em fluidos corporais. Eles refletem a distribuição da substância química ou seu metabólito através do organismo, e por esse motivo são classificados como dose interna, enquanto a dose externa se atribui à concentração do agente químico presente no ambiente em contato com o organismo. Alterações no perfil destes, auxiliam na elucidação de alterações morfológicas nos diversos órgãos (Z. Li et al., 2020).

Já os biomarcadores de efeito são alterações fisiológicas, bioquímicas, comportamentais ou outras em um organismo, que, dependendo da intensidade, pode ser reconhecida como estando relacionada a um dano possível ou instituído à saúde ou a uma doença. Enquanto os marcadores biológicos de suscetibilidade refletem fatores pré-existentes genéticos ou adquiridos que manipulam a resposta do organismo exposto a um certo xenobiótico, esses fatores independem da exposição e são denominados predominantemente genéticos (Silva, 2016). 


\subsection{Marcadores para diagnóstico de lesão hepática}

Os marcadores aspartato aminotransferase (AST) e alanina aminotransferase (ALT) são marcadores utilizados para o diagnóstico de lesões hepáticas. Tanto a AST quanto a ALT, poderá estar presente em outros tecidos como o tecido muscular. Entretanto, o fígado possui uma maior quantidade destas enzimas, sendo que a ALT encontraremos em maior quantidade no citoplasma dos hepatócitos e a AST será encontrada em sua maioria dentro de organelas citoplasmáticas (Castro, 2012) onde observamos um aumento de ALT em quadros de lesão aguda e AST em quadros de lesão crônica (de Moraes et al., 2011).

Por outro lado, marcadores como a proteína $\mathrm{C}$ reativa, a albumina e o perfil proteico irão auxiliar tanto no contexto inflamatório quanto na funcionalidade do fígado na síntese de proteínas e metabolismo. Uma redução drástica de albumina, é um forte indicativo de uma ausência de síntese hepática que poderá ser visto como uma possível lesão (Soares, 2013). Além disso, as lesões hepáticas estão em sua maioria associadas ao dano biliar, levando assim, a uma lesão hepatobiliar. Para as lesões biliares teremos os marcadores como a gama glutamil transferase (Gama GT) e a fosfatase alcalina como ponto de partida para avaliar a lesão dos ductos biliares (Gomes, 2014)

De acordo com estudos de casos e com os dados do The Fifth Medical Center of PLS General Hospital, Beijing, China, 2-11\% dos pacientes com COVID-19 tinham comorbidades hepáticas e 14-53\% dos casos relataram níveis anormais de ALT e AST durante a progressão da doença (Zhang et al., 2020). Ainda foi observado como alteração de destaque para pacientes infectados que a proteína $\mathrm{C}$ reativa obteve um aumento do valor em $60,7 \%$ e ocorreu a diminuição da concentração sérica de albumina causando comprometimento do fígado (Dutra, 2020). Por outro lado, foi observado que em pessoas com COVID-19, a função hepática teve alterações anormais estipulados como níveis elevados de alanina e aspartato aminotransferase, gama, glutamiltransferase fosfatase alcalina e bilirrubina total (Fan et al., 2020).

Segundo Gholizadeh et al., (2020), em um estudo feito em 279 pacientes que confirmaram COVID-19, e que tiveram os seus dados de marcadores bioquímicos hepáticos e hemograma completo definidos no primeiro dia da sua internação no hospital, demonstrou que a média do valor de lactato desidrogenase (LDH) de 621,29 U/L em todos os pacientes com COVID19 e a creatinofosfoquinase (CPK) foi de 286,90 U/L. A AST médio foi de 44,03 U/L em todos os pacientes, a ALT foi de 31,14 U/L. A relação AST / ALT foi de 1,64 em todos os pacientes. A dosagem da PCR aumentou em 79,93\% em todos os pacientes. Os valores médios de ALT e AST de pacientes com ALT elevada foram significativamente aumentados em comparação com pacientes com ALT normal (valor $\mathrm{P}=0,001$ ), enquanto a razão AST/ALT diminuiu significativamente em comparação com pacientes com ALT normal (valor P = 0,014). Além disso, o LDH médio de pacientes com ALT elevado foi significativamente aumentado em comparação com pacientes com ALT normal ( $\mathrm{P}$-valor = 0,014). Foi concluído que as lesões hepáticas e enzimas hepáticas anormais relacionadas à infecção por COVID-19 é uma alteração inflamatória aguda inespecífica (Gholizadeh et al., 2020).

Em outro estudo feito em 60 pacientes com COVID-19, 41 (69\%) pacientes tinham pelo menos uma alteração bioquímica hepática na admissão. A aspartato aminotransferase mediana (AST) foi maior do que a alanina aminotransferase (ALT) na admissão (46 vs. $30 \mathrm{U} / \mathrm{L}$ ) durante o curso hospitalar. As aminotransferases aumentaram acima do normal em 54 (93\%) pacientes, enquanto a fosfatase alcalina e as elevações da bilirrubina total foram raras. 10 (17\%) pacientes desenvolveram aminotransferases mais de 5 vezes o limite superior do normal. Foi comprovado que a AST está altamente correlacionada com ALT ao longo do curso da doença ( $r=0,97$; P < 0,0001) (I. B. S. da S. Costa et al., 2020; Bloom et al., 2020).

De acordo com uma revisão da literatura feita por Bertolini et al., (2020), 46\% dos pacientes internados com COVID19 tinham níveis elevados de aspartato aminotransferase (AST) plasmático e 35\% tinham níveis elevados de alanina aminotransferase (ALT) na admissão. As elevações de AST e ALT estão principalmente abaixo de 5 vezes o limite superior de referência e estão associadas à doença grave e aumento de marcadores inflamatórios. As elevações de AST e ALT são mais 
frequentes em pacientes norte-americanos do que em pacientes chineses. Elevações leves na gama-glutamiltransferase (GGT), fosfatase alcalina (ALP) e bilirrubina total também são relatadas, embora com menos frequência (Ten-Caten et al., 2020)

\subsection{Marcadores renais}

O principal metabólito nitrogenado que se deriva da degradação de proteínas pelo organismo é composto pela uréia, do qual 90\% desta substância é excretada pelos rins e aproximadamente 75\% desse montante corresponde ao nitrogênio nãoprotéico expelido, o restante é eliminado pela pele e pelo trato gastrintestinal (Sodré et al., 2007). A ureia é considerada como um preditor fraco da taxa de filtração glomerular (TFG), já que 40\% a 70\% voltam para o plasma por difusão passiva tubular, que depende do fluxo urinário. Desse modo a estase urinária aumenta o retorno da ureia ainda nos túbulos renais e a uma subestimação da TFG calculada pelo clareamento de ureia (Almeida, 2015). O principal uso clínico da ureia reside na determinação em conjunto com a creatinina. A razão uréia/creatinina sérica pode ser útil para avaliar pacientes com quedas bruscas de TFG (Dusse et al., 2017).

A creatinina é um produto residual proveniente da degradação da fosfocreatina oriunda do metabolismo muscular. Cerca de $98 \%$ da creatina permanece no músculo e $1,6 \%$ a $1,7 \%$ desta se transforma em creatinina diariamente, que é excretada pelo rim (Dusse et al., 2017). Portanto, a liberação e produção de creatina pelo músculo são basicamente constantes. Essa taxa de geração de creatinina é diretamente proporcional à massa muscular, variando de acordo com o sexo, etnia, e idade e é afetada por circunstancias que causam perda muscular (Soares, 2013). A creatinina é filtrada livremente pelo glomérulo e não é metabolizada nem absorvida pelo rim. No entanto, a quantidade de creatinina urinária que vem da secreção tubular é de aproximadamente $25 \%$, sendo está mais expressiva quanto menor for a taxa de filtração glomerular. A quantidade secretada é dependente da concentração sérica e do indivíduo, é inconstante, podendo ser alterada por efeitos de medicamentos (Dusse et al., 2017).

Apesar de suas principais características serem a insuficiência respiratória aguda e o dano alveolar difuso, o vírus causador do COVID-19 pode entrar no sangue, acumular-se nos rins e causar danos às células renais residentes (Huang et al., 2020). Interações como essas podem causar lesões renal aguda, que é caracterizada pela queda abrupta da TFG, aumento de creatinina sérica e produtos nitrogenados no sangue (Wang et al., 2020). O RNA de COVID-19 foi encontrado no plasma de $15 \%$ dos pacientes que apresentaram problemas renais por reação em cadeia da polimerase em tempo real (Huang et al., 2020). A ocorrência de lesão renal aguda em pacientes infectados pelo vírus SARS-CoV-2 é de cerca de 3 a 15\%, e em pacientes com infecção grave que requerem terapia intensiva em uma unidade de saúde, as taxas de lesão renal aguda aumentaram significativamente de 15\% para 50\% (Adapa et al., 2020). Para amenizar as lesões renais, os pacientes eram submetidos a hemodiálises, onde, no Brasil, o procedimento teve seu número quadruplicado (Jorge, 2020).

Estudos demonstraram níveis significativamente mais elevados de biomarcadores renais em pacientes infectados com COVID-19 (Xiang et al., 2020). Em uma pesquisa feita em 701 pacientes infectados com o vírus Sars-CoV-2, 43,9\% dos pacientes apresentaram proteinúria e 26,7\% apresentaram hematúria. Houve um aumento de creatinina sérica em $14.4 \%$, do nível de nitrogênio ureico no sangue em $13,1 \%$ a taxa de filtração glomerular foi estimada abaixo de $60 \mathrm{ml} / \mathrm{min}$ por $1.73 \mathrm{~m}^{2} \mathrm{em}$ $13,1 \%$ dos pacientes. Durante o período de estudo, 5,1\% dos pacientes desenvolveram lesão renal aguda. Os pacientes com creatinina sérica basal elevada apresentaram um aumento dos níveis de procalcitonina, aspartato aminotransferase, lactose desidrogenase, uma maior contagem de leucócitos e uma menor contagem de linfócitos e plaquetas, além de desenvolverem algumas anormalidades da via de coagulação, tais como, o tempo de tromboplastina parcial ativada prolongado e dímero D mais alto (Cheng et al., 2020). Eles também descobriram que esses pacientes eram mais propensos a necessitar de ventilação mecânica ou serem colocados em cuidados intensivos. Já em estudos realizados Kormann et al., (2020), onde ocorreu o acompanhamento de 42 pacientes, nos quais, após a infecção pelo SARS-COV-2, desenvolveram problemas renais, 
demonstraram a ocorrência de uma diferença no pico médio de creatinina sérica em pacientes na UTI com $181 \mu \mathrm{mol} / \mathrm{L}$ contra $82 \mu \mathrm{mol} / \mathrm{L}$ em pacientes internados. Esse aumento elevado de creatinina pode indicar insuficiência renal (SBN, 2020). A análise de regressão de Cox univariada revelou que níveis elevados de creatinina também estavam associados à mortalidade intra-hospitalar (Kermali et al., 2020).

De acordo com um estudo realizado em dois hospitais em Wuhan e um hospital em Huangshi em 193 pacientes infectados com COVID-19, 59\% dos pacientes apresentavam proteinúria, 44\% hematúria, 14\% tiveram um aumento nos níveis de nitrogénio ureico no sangue e $10 \%$ dos pacientes tiveram um aumento nos valores de creatinina sérica (Q. Li et al., 2020).

Qualquer efeito de proteinúria, inflamação ou lesão tubular na expressão tubular proximal de ACE2 ou na entrada viral de SARS-CoV-2 é atualmente desconhecido (Diao et al., 2020b). A replicação viral em podócitos e os danos decorrentes podem, em teoria, ser responsáveis pela proteinúria que foi relatada em pacientes com COVID-19 (Cheng et al., 2020). Além disso, a ativação de macrófagos hemofagocíticos associados à COVID-19 e a microangiopatia também podem causar lesão renal aguda e danos aos podócitos (Kermali et al., 2020).

\subsection{Marcadores cardíacos}

Os marcadores necessários para insuficiência cardíaca e de eventos coronários agudos são a Troponina T (TnT), peptídeo natriurético cerebral (BNP) e seu precursor (NT-proBNP) (Quiroga et al., 2012). A oportunidade de evitar um evento cardiovascular, tendo a descoberta precoce é o motivo principal para que exista motivação para as pesquisas, graças aos marcadores cardíacos, que são substâncias que são liberadas no sangue quando há alguma lesão cardíaca (Chen et al., 2020). Os marcadores são utilizados para diagnosticar, monitorar e avaliar os pacientes com suspeita de síndrome coronariana aguda, assim tendo a prevenção primária para essa doença (Doron \& Munoz, 2015).

A isoenzima MB é uma opção adequada, especialmente se a dosagem de uma das troponinas não estiver disponível. Esta isoenzima possui elevada sensibilidade e especificidade para o diagnóstico de lesão do músculo cardíaco (Guo det al., 2020). Por outro lado, as troponinas são proteínas estruturais envolvidas no processo de contração das fibras musculares esqueléticas e cardíacas. O complexo troponina é composto por três proteínas: troponina T, troponina I e troponina C. Como existem diferenças antigênicas entre as troponinas dos músculos esqueléticos e cardíacos, o uso de anti-soros específicos permite a identificação e quantificação de cada uma delas. As troponinas T (cTnT) e I (cTnI) são consideradas como os marcadores bioquímicos mais específicos e sensíveis para o diagnóstico de lesão isquêmica do miocárdio (Cao et al., 2020).

De acordo com os dados da sociedade brasileira de cardiologia (SBC), em março de 2020, ao analisar o acesso cardiovascular que foi relacionado ao novo vírus (COVID 19) foi apresentado a conclusão; arritmias (16\%), miocardite (7,2\%), choque (1-2\%), isquemia miocárdica (10\%). E com isso concluído a possibilidade de termos a COVID-19 como fonte de miocardite aguda (Rente et al., 2020).

Segundo estudos, uma gama de complicações cardíacas está associadas aos pacientes com COVID-19. Na qual se verificou condições cardiovasculares subjacentes, às elevações de troponina cardíaca I de alta sensibilidade; N-terminal do pró hormônio do peptídeo natriurético do tipo B e creatina quinase-MB; e as complicações cardiovasculares foram associadas ao aumento do risco de óbito com a infecção por SARS-CoV-2 (Martins-Filho et al., 2020).

Os danos ao sistema cardiovascular decorrente da COVID-19 ocorrem principalmente nos pacientes com fatores de risco cardiovascular (idade avançada, hipertensão e diabetes) ou com DCV prévia. A resposta inflamatória que foi gerada com essa infecção viral leva a lesão do sistema cardiovascular e pulmões com aumento e elevação de dímero-D, procalcitonina, proteína $\mathrm{C}$ reativa, ferritina, troponina e NT-proBNP, e que culmina em complicações cardiovasculares e óbito. Observaram também concentrações mais altas de citocinas que estão relacionadas ao agravo do sistema cardiovascular (Cheng et al., 2020). 
Além de todos os marcadores inflamatórios citados, nos pacientes com COVID-19 observa-se também os marcadores de disfunção miocárdica, o aumento nos níveis de BNP e NT-proBNP. Reforçando assim que aqueles que apresentam afronte miocárdica estão dispostos a desenvolver problemas na função cardíaca, como IC, miocardite, infarto agudo do miocárdio, choque e arritmias (Costa et al., 2020)

A lesão cardíaca, indicada pelo aumento da troponina I hipersensível (hs-cTnI), é uma comorbidade comum em pacientes com COVID-19, relatada em 12 a 77\% dos casos (Chen et al., 2020). Em outro estudo foi observado entre 41 pacientes hospitalizados inscritos a lesão cardíaca aguda foi de $5(12 \%)$ casos. A hs-cTnI elevada também foi associada à morte (Huang et al., 2020).

Ao analisar níveis de CKMB e troponina em pacientes, Shi et al., (2020), identificou que 82 de 416 (19,7\%) pacientes com COVID-19 apresentaram lesão miocárdica, diagnosticada por níveis séricos de cTnI significativamente elevados. Ocorrendo uma taxa de mortalidade significativamente maior de 51,2\% em comparação com uma mortalidade de $4,5 \%$ em pacientes com níveis normais de cTnI e sem lesão miocárdica, expondo a gravidade de lesão miocárdica em pacientes com COVID-19. Já Wang et al., 2020, analisou que 36 de 138 (26,1\%) pacientes com COVID-19 estavam na UTI com graves sintomas, todos com níveis séricos de cTnI e CK-MB significativamente elevados ( $\mathrm{p}=0,004$ ep <0,001, respectivamente) em comparação com pacientes fora da UTI.

\subsection{Marcadores pancreáticos}

A amilase é uma enzima que pertence à classe das hidrolases, ela é responsável por catalisar a hidrólise de amido e glicogênio ingeridos na dieta. A amilase sérica é principalmente excretada pelas glândulas salivares e pelas células acinares do pâncreas (Rodrigues, 2018). A amilase chega ao duodeno por meio do ducto pancreático, no intestino ela hidrolisa açúcares, carboidratos e seus componentes. Se as células acinares do pâncreas sofrerem uma lesão (pancreatite) ou se o fluxo do ducto pancreático for obstruído (carcinoma pancreático) a amilase irá fluir para o sistema linfático intra-pancreático e peritônio e, por drenagem, irá atingir os vasos sanguíneos (Rodrigues, 2018). A amilase sérica apresenta-se aumentada nas pancreatites agudas e crônicas e os valores baixos de amilase podem indicar insuficiência pancreática ou doenças graves no fígado (Telli et al., 2016).

A Lipase é uma enzima hidrolase que tem como função principal catalisar a hidrólise de gorduras e óleos liberando ácidos graxos, diacilgliceróis, monoacilgliceróis e glicerol (Muruci et al., 2001). A lipase se encontra em baixas quantidades no sangue em um indivíduo saudável, mas quando o pâncreas apresenta um mau funcionamento, esse volume acaba aumentando para que as condições favoráveis ao organismo sejam retomadas. Monitorar o nível de lipase no sangue é essencial para se diagnosticar processos inflamatórios no aparelho digestivo (Marchioro, 2012). A lipase é um marcador mais específico de doença pancreática aguda do que a amilase. No entanto, devido ao comprometimento da resposta imune e a capacidade pancreática pode haver inflamação crônica quando auxiliado com COVID-19 (McNabb-Baltar et al., 2020).

De acordo com um estudo realizado em 71 pacientes hospitalizados pelo COVID-19 de 23 de janeiro de 2020 a 2 de abril de 2020, em 6 hospitais em Massachusetts, 9 desenvolveram aumento de lipase, com 2 a 3 vezes o limite superior do normal. Nenhum paciente desenvolveu pancreatite aguda. Por se ligarem aos receptores ACE2 presentes no pâncreas, o SARSCOV-2 faz com que ocorra um processo inflamatório no local, não necessariamente levando a uma pancreatite aguda, mas causando o aumento de enzimas como lipase e amilase (Paravidino et al., 2020).

Em outro estudo feito por Wang et al., (2020) com 52 pacientes infectados com o vírus SARS-CoV-2, Nove (17\%) pacientes tiveram pancreatite aguda, apresentando amilase $(115 \pm 25)$ e Lipase $(71 \pm 34)$ altas. Esses resultados mostram padrões potenciais de lesão pancreática leve em pacientes com COVID-19, e podem estar relacionados ao envolvimento viral direto do pâncreas ou de anormalidades enzimáticas secundárias no contexto de doença grave sem lesão pancreática 
substancial. Eles não mostram pancreatite clinicamente grave como uma manifestação comum. Pesquisas adicionais e séries maiores são necessárias para avaliar se um subconjunto de pacientes tem pancreatite clínica como entidade patológica presente ou concomitante.

\section{Conclusão}

Com o presente estudo, conclui-se que o COVID-19 não causa apenas problemas relacionados aos órgãos respiratórios, como se imaginava no início, e sim problemas sistemáticos. Problemas renais estão entre os problemas mais frequentes em pacientes com COVID-19, mas também ocorre problemas hepáticos, pancreáticos, cardíacos e entre outros, nos quais, as alterações de seus marcadores bioquímicos podem se tornar uma forma de rastrear o agravamento dos quadros clínicos antes mesmo de acontecer. Sendo assim, os resultados obtidos abrem margem para a pesquisa e rastreio laboratorial dos marcadores que mais se alteram em quadros de COVID-19, podendo auxiliar no prognóstico de pacientes em quadros graves da doença.

\section{Agradecimentos}

A Universidade José do Rosário Vellano e cursos de Biomedicina e Farmácia.

\section{Referências}

Adapa, S., Aeddula, N. R., Konala, V. M., Chenna, A., Naramala, S., Madhira, B. R., Gayam, V., Balla, M., Muppidi, V., \& Bose, S. (2020). COVID-19 and Renal Failure: Challenges in the Delivery of Renal Replacement Therapy. Journal of Clinical Medicine Research, 12(5), 276-285. https://doi.org/10.14740/jocmr4160

Almeida, L. salles de. (2015). Sildenafil previne a nefropatia induzida por contraste em ratos wistar.

Amorim, L. C. A. (2003). Os biomarcadores e sua aplicação na avaliação da exposição aos agentes químicos ambientais. Revista Brasileira de Epidemiologia, 6(2), 158-170. https://doi.org/10.1590/s1415-790x2003000200009

Bertolini, A., van de Peppel, I. P., Bodewes, F. A. J. A., Moshage, H., Fantin, A., Farinati, F., Fiorotto, R., Jonker, J. W., Strazzabosco, M., Verkade, H. J., \& Peserico, G. (2020). Abnormal Liver Function Tests in Patients With COVID-19: Relevance and Potential Pathogenesis. Hepatology, 72(5), 1864-1872. https://doi.org/10.1002/hep. 31480

Bloom, P. P., Meyerowitz, E. A., Reinus, Z., Daidone, M., Gustafson, J., Kim, A. Y., Schaefer, E., \& Chung, R. T. (2020). Liver Biochemistries in Hospitalized Patients With COVID-19. Hepatology, O(0), 1-11. https://doi.org/10.1002/hep.31326

Cao, J., Zheng, Y., Luo, Z., Mei, Z., Yao, Y., Liu, Z., Liang, C., Yang, H., Song, Y., Yu, K., Gao, Y., Zhu, C., Huang, Z., Qian, J., \& Ge, J. (2020). Myocardial injury and COVID-19: Serum hs-cTnI level in risk stratification and the prediction of 30-day fatality in COVID-19 patients with no prior cardiovascular disease. Theranostics, 10(21), 9663-9673. https://doi.org/10.7150/thno.47980

Castro, M. (2012). Exames Laboratoriais Conteúdo.

Chen, C., Li, H., Hang, W., \& Wang, D. W. (2020). Cardiac injuries in coronavirus disease 2019 (COVID-19). Journal of Molecular and Cellular Cardiology, 145(May), 25-29. https://doi.org/10.1016/j.yjmcc.2020.06.002

Cheng, Y., Luo, R., Wang, K., Zhang, M., Wang, Z., Dong, L., Li, J., Yao, Y., Ge, S., \& Xu, G. (2020). Kidney disease is associated with in-hospital death of patients with COVID-19. Kidney International, 97(5), 829-838. https://doi.org/10.1016/j.kint.2020.03.005

Costa, I. B. S. da S., Bittar, C. S., Rizk, S. I., Filho, A. E. de A., Santos, K. A. Q., Machado, T. I. V., Andrade, F. T. de A., González, T. B., Arévalo, A. N. G., de Almeida, J. P., Bacal, F., de Oliveira, G. M. M., de Lacerda, M. V. G., Barberato, S. H., Chagas, A. C. P., Rochitte, C. E., Ramires, J. A. F., Filho, R. K., \& Hajjar, L. A. (2020). The heart and COVID-19: What cardiologists need to know. Arquivos Brasileiros de Cardiologia, 114(5), 805-816. https://doi.org/10.36660/abc.20200279

Croda, J. H. R., \& Garcia, L. P. (2020). Resposta imediata da Vigilância em Saúde à epidemia da COVID-19. Epidemiologia e Servicos de Saude : Revista Do Sistema Unico de Saude Do Brasil, 29(1), e2020002. https://doi.org/10.5123/S1679-49742020000100021

Cui, H., Wu, F., Fan, Z., Cheng, X., Cheng, J., \& Fan, M. (2020). The effects of renin-angiotensin system inhibitors (RASI) in coronavirus disease (COVID19) with hypertension: A retrospective, single-center trial. Medicina Clínica (English Edition), 155(7), 295-298. https://doi.org/10.1016/j.medcle.2020.06.007

da Costa, V. G., Moreli, M. L., \& Saivish, M. V. (2020). The emergence of SARS, MERS and novel SARS-2 coronaviruses in the 21 st century. Archives of Virology, 165(7), 1517-1526. https://doi.org/10.1007/s00705-020-04628-0

de Moraes, T. P., Fortes, P. C. N., Ribeiro, S. C., Riella, M. C., \& Pecoits-Filho, R. (2011). Comparative analysis of lipid and glucose metabolism biomarkers in non-diabetic hemodialysis and peritoneal dialysis patients. Jornal Brasileiro de Nefrologia : 'orgão Oficial de Sociedades Brasileira e Latino-Americana de 
Nefrologia, 33(2), 173-179. https://doi.org/10.1590/s0101-28002011000200009

de Paula Antunes, B. B., Peres, I. T., Baião, F. A., Ranzani, O. T., dos Santos Lourenço Bastos, L., de Araújo Batista da Silva, A., de Souza, G. F. G., Marchesi, J. F., Dantas, L. F., Vargas, S. A., Maçaira, P., Hamacher, S., \& Bozza, F. A. (2020). Progression of confirmed COVID-19 cases after the implementation of control measures. Revista Brasileira de Terapia Intensiva, 32(2), 213-223. https://doi.org/10.5935/0103-507X.20200028

Diao, B., Wang, C., Wang, R., Feng, Z., Tan, Y., Wang, H., Wang, C., Liu, L., Liu, Y., Liu, Y., Wang, G., Yuan, Z., Ren, L., Wu, Y., \& Chen, Y. (2020a). Human kidney is a target for novel severe acute respiratory syndrome coronavirus 2 (SARS-CoV-2) infection. MedRxiv, 1380-1383. https://doi.org/10.1101/2020.03.04.20031120

Diao, B., Wang, C., Wang, R., Feng, Z., Tan, Y., Wang, H., Wang, C., Liu, L., Liu, Y., Liu, Y., Wang, G., Yuan, Z., Ren, L., Wu, Y., \& Chen, Y. (2020b). Human Kidney is a Target for Novel Severe Acute Respiratory Syndrome Coronavirus 2 (SARS-CoV-2) Infection. 1380-1383. https://doi.org/10.1101/2020.03.04.20031120

Doron, D., \& Munoz, M. (2015). Marcadores Cardíacos Y Riesgo Cardiovascular. Revista de Medicina Clínica CONDES, 26(2), $133-141$.

Dusse, L. M. S., Rios, D. R. A., Sousa, L. P. N., Moraes, R. M. M. e S., Domingueti, C. P., \& Gomes, K. B. (2017). Biomarkers of Renal Function: What Is Currently Available? Revista Brasileira de Análises Clínicas, 49(1), 1-15. https://doi.org/10.21877/2448-3877.201600427

Dutra, D. (2020). Novo Coronavírus Covid-19 (SARS-CoV-2). InviNews, 19(1).

Fan, Z., Chen, L., Li, J., Cheng, X., Yang, J., Tian, C., Zhang, Y., Huang, S., Liu, Z., \& Cheng, J. (2020). PANCREAS , BILIARY TRACT , AND LIVER Clinical Features of COVID-19-Related Liver Functional Abnormality. Clinical Gastroenterology and Hepatology, 18(7), 1561-1566. https://doi.org/10.1016/j.cgh.2020.04.002

Gholizadeh, P., Safari, R., Zeinalzadeh, E., Pagliano, P., Ganbarov, K., Esposito, S., \& Khodadadi, E. (2020). Alteration of Liver Biomarkers in Patients with . $285-292$.

Gomes, D. L. F. (2014). Biomarcadores para Avaliação da Lesão Hepática Induzida por Fármacos. https://sapientia.ualg.pt/handle/10400.1/8027

Huang, C., Wang, Y., Li, X., Ren, L., Zhao, J., Hu, Y., Zhang, L., Fan, G., Xu, J., Gu, X., Cheng, Z., Yu, T., Xia, J., Wei, Y., Wu, W., Xie, X., Yin, W., Li, H., Liu, M., \& Cao, B. (2020). Clinical features of patients infected with 2019 novel coronavirus in Wuhan, China. The Lancet, 395(10223), 497-506. https://doi.org/10.1016/S0140-6736(20)30183-5

Jia, H. P., Look, D. C., Shi, L., Hickey, M., Pewe, L., Netland, J., Farzan, M., Wohlford-Lenane, C., Perlman, S., \& McCray, P. B. (2005). ACE2 Receptor Expression and Severe Acute Respiratory Syndrome Coronavirus Infection Depend on Differentiation of Human Airway Epithelia. Journal of Virology, 79(23), 14614-14621. https://doi.org/10.1128/jvi.79.23.14614-14621.2005

Kermali, M., Khalsa, R. K., Pillai, K., Ismail, Z., \& Harky, A. (2020). The role of biomarkers in diagnosis of COVID-19 - A systematic review. Life Sciences, 254(May), 117788. https://doi.org/10.1016/j.1fs.2020.117788

Kormann, R., Jacquot, A., Alla, A., Corbel, A., Koszutski, M., Voirin, P., Garcia Parrilla, M., Bevilacqua, S., Schvoerer, E., Gueant, J.-L., Namour, F., Levy, B., Frimat, L., \& Oussalah, A. (2020). Coronavirus disease 2019: acute Fanconi syndrome precedes acute kidney injury. Clinical Kidney Journal, 13(3), 362370. https://doi.org/10.1093/ckj/sfaa109

Li, Q., Cao, Y., Chen, L., Wu, D., Yu, J., Wang, H., He, W., Chen, L., Dong, F., Chen, W., Chen, W., Li, L., Ran, Q., Liu, Q., Ren, W., Gao, F., Chen, Z., Gale, R. P., \& Hu, Y. (2020). Hematological features of persons with COVID-19. Leukemia, 34(8), 2163-2172. https://doi.org/10.1038/s41375-020-0910-1

Li, Z., Wu, M., Yao, J., Guo, J., Liao, X., Song, S., Li, J., Duan, G., Zhou, Y., Wu, X., Zhou, Z., Wang, T., Hu, M., Chen, X., Fu, Y., Lei, C., Dong, H., Xu, C., Hu, Y., \& Yan, J. (2020). Kidney Dysfunctions of COVID-19 Patients: A Multi-Centered, Retrospective, Observational Study. SSRN Electronic Journal. https://doi.org/10.2139/ssrn.3556634

Loeffelholz, M. J., \& Tang, Y. W. (2020). Laboratory diagnosis of emerging human coronavirus infections-the state of the art. Emerging Microbes and Infections, 9(1), 747-756. https://doi.org/10.1080/22221751.2020.1745095

Marchioro, A. (2012). Efeitos Das Aflatoxinas No Pâncreas Exócrino E Desempenho De Frangos De Corte.

Martins-Filho, P. R., Barreto-Filho, J. A. S., \& Santos, V. S. (2020). Myocardial injury biomarkers and cardiac complications associated with mortality in patients with covid-19. Arquivos Brasileiros de Cardiologia, 115(2), 273-277. https://doi.org/10.36660/abc.20200372

McNabb-Baltar, J., Jin, D. X., Grover, A. S., Redd, W. D., Zhou, J. C., Hathorn, K. E., McCarty, T. R., Bazarbashi, A. N., Shen, L., \& Chan, W. W. (2020). Lipase Elevation in Patients With COVID-19. The American Journal of Gastroenterology, 115(8), 1286-1288. https://doi.org/10.14309/ajg.0000000000000732

Muruci, L. N. M., Santos, R. R. Dos., Couri, S., Penha, E. D. M., \& Damaso, M. C. T. (2001). Estudo das características físicos-químicas de lipase de.

Paravidino, L. M., Valencia, H. C. M., Barreto, L. O., Wekid, M. L. F., Almeida, M. R., \& Valencia, M. M. M. (2020). Covid-19 Associada a Pancreatite Aguda: Um Relato De Caso. Revista Científica Da Faculdade de Medicina de Campos, 15(2), 35-38. https://doi.org/10.29184/19807813.rcfmc.395.vol.15.n2.2020

Quiroga, B., Goicoechea, M., García De Vinuesa, S., Verde, E., Verdalles, Ú., Yuste, C., Reque, J., \& Luño, J. (2012). Marcadores cardiacos en diferentes grados de enfermedad renal crónica: influencia de la inflamación y de la afectación cardiaca previa. Medicina Clinica, 139(3), 98-102. https://doi.org/10.1016/j.medcli.2011.05.014

Rente, A., Junior, D. U., \& Uezato, K. M. K. (2020). ABC Cardiol. Abc Cardiol, 113(2), 305 p.

Rodrigues, D. (2018). Bioquímica p/ EBSERH (Biomédico) Teoria e exercícios comentados. 
Research, Society and Development, v. 10, n. 3, e6310313045, 2021

(CC BY 4.0) | ISSN 2525-3409 | DOI: http://dx.doi.org/10.33448/rsd-v10i3.13045

Serrano-Cumplido, A., Antón-Eguía Ortega, P. B., Ruiz García, A., Olmo Quintana, V., Segura Fragoso, A., Barquilla Garcia, A., \& Morán Bayón. (2020). COVID-19. History repeats itself and we keep stumbling on the same stone. Semergen, 46, 48-54. https://doi.org/10.1016/j.semerg.2020.06.008

Shi, S., Qin, M., Shen, B., Cai, Y., Liu, T., Yang, F., Gong, W., Liu, X., Liang, J., Zhao, Q., Huang, H., Yang, B., \& Huang, C. (2020). Association of Cardiac Injury with Mortality in Hospitalized Patients with COVID-19 in Wuhan, China. JAMA Cardiology, 5(7), 802-810. https://doi.org/10.1001/jamacardio.2020.0950

Silva, A. C. de A. (2016). Biomarcadores de Contaminação Ambiental. https://bdigital.ufp.pt/bitstream/10284/5821/1/PPG_29729.pdf

Soares, A. A. (2013). Ferramentas Para Detecção Da Doença Renal: Valores De Referência Da Taxa De Filtração Glomerular E Desempenho Das Equações De Estimativa Com Creatinina E Cistatina C Séricas Em Indivíduos Saudáveis. Universidade Federal do Rio Grande do Sul.

Sodré, F. L., Costa, J. C. B., \& Lima, J. C. C. (2007). Avaliação da função e da lesão renal: Um desafio laboratorial. Jornal Brasileiro de Patologia e Medicina Laboratorial, 43(5), 329-337. https://doi.org/10.1590/s1676-24442007000500005

Telli, E. M. R. P., Frigeri, M., \& Mello, S. R. (2016). Avaliação da atividade de enzimas hepáticas em dependentes, ex-dependentes e não usuários do etanol Evaluation of hepatic enzyme activity in dependent, ex-dependent and non-users of ethanol. Brazilian Journal of Clinical Analysis, 48(3), 245-252. http://www.rbac.org.br/wp-content/uploads/2016/11/ARTIGO-10_RBAC-48-3-2016-ref.-188.pdf

ten-Caten, F., Gonzalez-Dias, P., Castro, Í, Ogava, R. L. T., Giddaluru, J., Silva, J. C. S., Martins, F., Gonçalves, A. N. A., Costa-Martins, A. G., Araujo, J. D., Viegas, A. C., Cunha, F. Q., Farsky, S., Bozza, F. A., Levin, A. S., Pannaraj, P. S., de Silva, T. I., Minoprio, P., Andrade, B. B., \& Nakaya, H. I. (2020) In-depth Analysis of Laboratory Parameters Reveals the Interplay between Sex, Age and Systemic Inflammation in Individuals with COVID-19. MedRxiv, 123. https://doi.org/10.1101/2020.08.07.20170043

Wang, D., Hu, B., Hu, C., Zhu, F., Liu, X., Zhang, J., Wang, B., Xiang, H., Cheng, Z., Xiong, Y., Zhao, Y., Li, Y., Wang, X., \& Peng, Z. (2020). Clinical Characteristics of 138 Hospitalized Patients with 2019 Novel Coronavirus-Infected Pneumonia in Wuhan, China. JAMA - Journal of the American Medical Association, 323(11), 1061-1069. https://doi.org/10.1001/jama.2020.1585

Xiang, J., Wen, J., Yuan, X., Xiong, S., Zhou, X., Liu, C., \& Min, X. (2020). Potential biochemical markers to identify severe cases among COVID-19 patients. 19, 1-10. https://doi.org/10.1101/2020.03.19.20034447

Yu, J. (2020). From Mutation Signature to Molecular Mechanism in the RNA World: A Case of SARS-CoV-2. Genomics, Proteomics and Bioinformatics, xxxx. https://doi.org/10.1016/j.gpb.2020.07.003

Yuki, K., Fujiogi, M., \& Koutsogiannaki, S. (2020). Since January 2020 Elsevier has created a COVID-19 resource centre with free information in English and Mandarin on the novel coronavirus COVID- 19. The COVID-19 resource centre is hosted on Elsevier Connect, the company 's public news and information . Clinical Immunology, January.

Zhang, H., Liao, Y. S., Gong, J., Liu, J., Xia, X., \& Zhang, H. (2020). Clinical characteristics of coronavirus disease (COVID-19) patients with gastrointestinal symptoms: A report of 164 cases. Digestive and Liver Disease, 52(10), 1076-1079. https://doi.org/10.1016/j.dld.2020.04.034 